\title{
Artificial magnetic metamaterial design by using spiral resonators
}

\author{
Juan D. Baena, ${ }^{*}$ Ricardo Marqués, ${ }^{\dagger}$ and Francisco Medina ${ }^{\ddagger}$ \\ Departamento de Electrónica y Electromagnetismo, Facultad de Física, Universidad de Sevilla, Avenida Reina Mercedes s/n, \\ 41012 Sevilla, Spain \\ Jesús Martel ${ }^{\S}$ \\ Departamento de Física Aplicada II, ETS de Arquitectura, Universidad de Sevilla, Avenida Reina Mercedes $s / n$, \\ 41012 Sevilla, Spain \\ (Received 25 June 2003; published 8 January 2004)
}

\begin{abstract}
A metallic planar particle, that will be called spiral resonator (SR), is introduced as a useful artificial atom for artificial magnetic media design and fabrication. A simple theoretical model which provides the most relevant properties and parameters of the SR is presented. The model is validated by both electromagnetic simulation and experiments. The applications of SR's include artificial negative magnetic permeability media (NMPM) and left-handed-media (LHM) design. The main advantages of SR's for such purpose are small electrical size at resonance, absence of magnetoelectric coupling (thus avoiding bianisotropic effects in the continuous medium made of these particles), and easy fabrication. Experimental confirmation of NMPM and LHM behavior using SR's is also reported.
\end{abstract}

DOI: 10.1103/PhysRevB.69.014402

PACS number(s): 42.70.Qs, 41.20.Jb, 42.25.Bs, 73.20.Mf

\section{INTRODUCTION}

In 1999 Pendry et al. ${ }^{1}$ proposed an artificial negative magnetic permeability medium (NMPM) composed of a regular array of electrically small resonant particles referred as split ring resonators (SRR). These particles show both small electrical size and strong magnetic polarizability at resonance, the particle being diamagnetic above such resonance. Later, Smith and co-workers ${ }^{2}$ validated this idea and manufactured the first left-handed-medium (LHM) following Veselago's terminology ${ }^{3}$ - by means of the superposition of the reported NMPM and a regular array of conducting wires responsible for the negative permittivity (the wires array behaves as a low loss artificial plasma ${ }^{4}$ having negative permittivity below its plasma frequency). In fact, it has been shown ${ }^{5}$ that the SRR is a magnetoelectric particle thus making the LHM in Ref. 2 to be a bianisotropic material. A modified version of the SRR that does not present magnetoelectric coupling-the so-called modified or broadside coupled SRR (BC-SRR)—was introduced in Ref. 5. Moreover, some of the authors have recently shown that the artificial plasma behavior can be obtained by replacing the array of wires by a hollow pipe metallic waveguide. This idea leads to a one-dimensional simulation of a LHM by using a SRR-loaded waveguide. ${ }^{6}$ The same concept has been then extended to the simulation and design of an isotropic two-dimensional LHM. ${ }^{7}$

On the other hand, planar spirals are well-known structures in microwave engineering, where they are commonly used as lumped inductors, ${ }^{8}$ usually in the presence of a ground plane. However, as far as we know, they have never been used in metamaterial design. The aim of this paper is to explore this last application of planar spiral resonators (SR's). It will be shown that the use of SR's provides a potential reduction of the electrical size of the metamaterial unit cell. This reduction is crucial if the metamaterial has to be viewed as a continuous medium. In fact, to the best of our knowledge, all the reported LHM show unit cell sizes higher than one-tenth of the free space wavelength, in the very limit of application of the continuous medium description. Moreover, the SR's are not bianisotropic particles, having an easier fabrication process than the previously reported alternative to get that feature, the BC-SRR's (SR is a uniplanar particle, while BC-SRR requires accurate alignment of metal pattern at two sides of a dielectric substrate). Thus SR's can be advantageously used in the design and fabrication of NMPM or LHM. This will be also experimentally shown in this paper.

\section{MODELS FOR THE SRR AND THE SR}

The particles analyzed and compared in this paper are represented in Fig. 1. The SRR is formed by two coupled conducting rings printed on a dielectric slab, while the two and three turns spiral resonators (SR2 and SR3) are made by a single strip rolled up to form a spiral. The SRR has been already analyzed by some of the authors in Ref. 5 and 9 . This analysis shows that, assuming a particle size much smaller than the free space wavelength, the SRR's essentially behaves as a quasistatic $L C$ circuit fed by the external magnetic flux linked by the particle. The total current in the circuit, i.e., the sum of the currents on each ring for a given value of the angular polar coordinate $\phi$ is uniform (it does not depend on $\phi$ ), since the current lines go from one ring to other across the slot between the rings in the form of field displacement current lines. Thus, assuming that the capacitance between contiguous rings is much larger than the slit capacitance, the circuit model of Fig. 1(a) is perfectly justified. Note that the proper circuit capacitance is the series connection of the capacitances of the upper and lower halves of the SRR. This analysis will be now applied to the SR's considered in this paper. The hypothesis about how the conduction current distributes on each ring is schematically shown, once again, in Fig. 1(a)-1(c). Note that, in general, the conduction 
a)

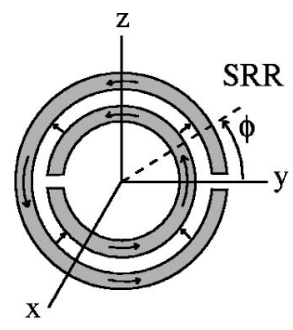

b)

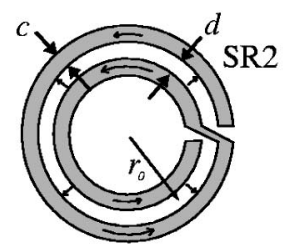

c)

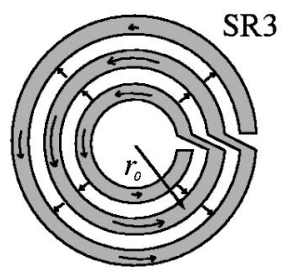

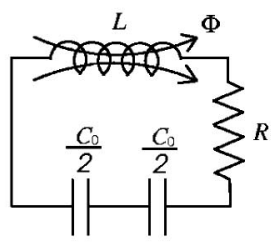
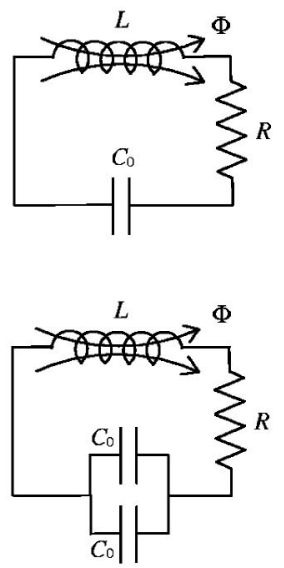
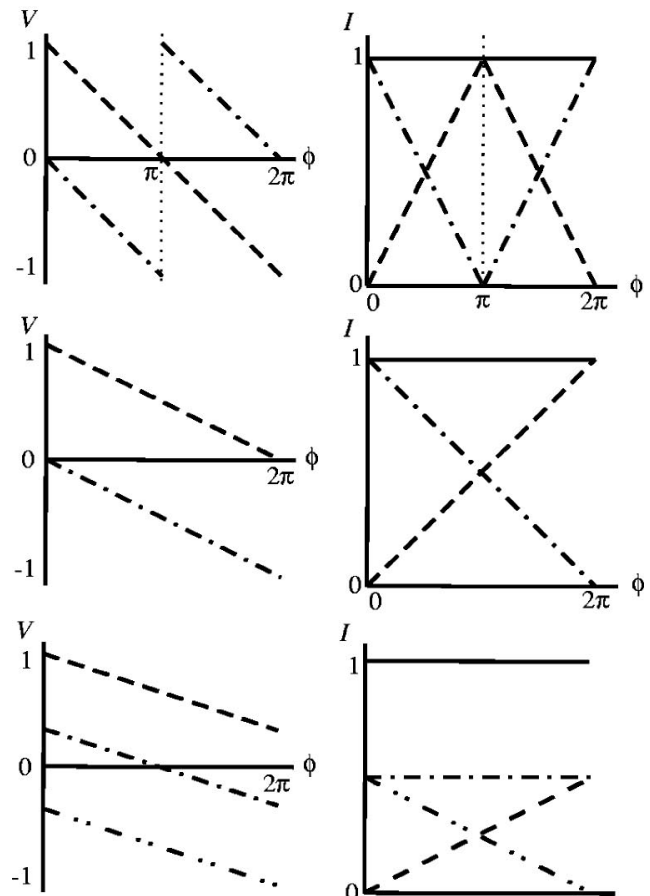

FIG. 1. In each row of this figure it has been represented the top view of a resonant magnetic particle (SRR, SR2, and SR3), its equivalent circuit, the normalized quasistatic voltage as a function of the angular polar coordinate $V(\phi)$ and the normalized electric current intensity $I(\phi)$ along the strips. The dashed lines in the graphics for both $V$ and $I$ (normalized to the highest value) correspond to the first (- - ), second (- - ) and, eventually, third (- - - ), ring (counting from the outer to the inner). The continuous line in the $I$ curves stands for the total current in the particle, i.e., the sum of all the two (or three) dashed lines. (a) Split ring resonator (SRR), (b) two turns spiral resonator (SR2), and (c) three turns spiral resonator (SR3). All particles have the same average radius of the rings $r_{0}$.

current intensity varies along the metal strips (quasilinearly with the angular coordinate $\phi$ in the low-frequency limit, except for the case of the middle ring of SR3, where the current distribution is roughly uniform with $\phi$ ). If small parasitic capacitances (slit capacitance in the SRR, open-end capacitances in any of the resonators, or the capacitance between nonadjacent strips in the case of SR3) are neglected, the conduction current intensity must vanish at the ends of each metallic strip, as it is represented in the current curves in Fig. 1. Quasistatic voltage distribution helps us to identify which capacitors are involved in the equivalent circuit model of each of the structures and how they are connected. Indeed, each configuration has a different associated overall capacitance, and this is what makes them different from the point of view adopted in this paper. Inductance, however, can be considered roughly the same for all the three structures if similar dimensions are assumed, since global conduction current flow (the sum of the contributions of the rings for each particular value of $\phi$ ) is uniform. The inductance $L$ in such case, can be approximated by the magnetostatic inductance of a single ring of width $c$ and mean radius equal to the average radius of the particle. Once the suitable capacitance is determined for each of the particles under study, the resonance frequency will be given by

$$
\omega_{0}^{2}=\frac{1}{L C}
$$

where $L$ is the aforementioned inductance and $C$ is the capacitance of the proper equivalent resonant circuit. Following the rationale proposed in Ref. 9 for the SRR - as it has been done at the beginning of this section - or just looking at the voltage distribution shown in Fig. 1(a), it is obvious that each of the halves of that particle contributes to the global capacitance in the form of a series connection of two capacitors, each of value $C_{0} / 2$, where $C_{0}=2 \pi r_{0} C_{\text {pul }}$ is the capacitance between two ring shaped electrodes. In this expression $C_{\text {pul }}$ is the per unit length capacitance between two straight metal strips having the same width and separation than the ones forming part of the coupled ring structure. Curvature effects are ignored, but we are just looking for an approximate solution. Fine adjustments can be made using electromagnetic simulation software if desired (although, as we will see later, the approximate results are quite satisfactory). The situation for SR2 is slightly different. From the current and voltage distributions depicted in Fig. 1(b), it is clear that $C_{0}$ is the capacitance involved in the equivalent resonator. For the third particle, SR3, we can approximately say that the relevant capacitance is, roughly speaking, $2 C_{0}$, assuming that we ignore the difference between the capacitances of the pair of external and internal rings. These two capacitances are parallel connected as we can easily deduce from the voltage distribution depicted in Fig. 1(c). We can, once again, perform a more accurate analysis by using a commercial electromagnetic simulator or home-made software to accu- 
rately compute the exact values of the capacitances, but this only would provide the fine adjustment. We can now summarize our results for the capacitances of the three particles in Fig. 1 as follows:

$$
C_{\mathrm{SRR}} \approx C_{0} / 4, \quad C_{\mathrm{SR} 2} \approx C_{0}, \quad C_{\mathrm{SR} 3} \approx 2 C_{0} .
$$

Explicit working expressions for $L$ and $C_{\text {pul }}$ are given in Ref. 9, but any method for computing the quasistatic capacitances and inductances could be used. More interestingly, this analysis predicts the following relation among the resonance frequencies of the different particles:

$$
f_{0}^{\mathrm{SRR}} \approx 2 f_{0}^{\mathrm{SR} 2} \approx 2 \sqrt{2} f_{0}^{\mathrm{SR} 3} .
$$

Finally, ohmic losses can be taken into account by including the resistance of the rings in the equivalent circuit ${ }^{9}$ (note that skin effect must be accounted for).

The previously mentioned potential reduction of the electrical size of the metamaterial unit cell by using SR's (instead of SRR's) is directly deduced from the rule (3), which can be reformulated for the electrical size at resonance $s_{0}$ as $s_{0}^{\mathrm{SRR}} \approx 2 s_{0}^{\mathrm{SR} 2}$. For the SR3 this reduction is not directly given by Eq. (3), since for a SRR and a SR3 with the same mean radius, the external radius of the SR3 is larger than the external radius of the SRR (see Fig. 1). Thus, the electrical size is reduced by a factor slightly higher than $1 /(2 \sqrt{2})$. It must be remembered that the SRR particle shows a magnetoelectric coupling between the electric and magnetic
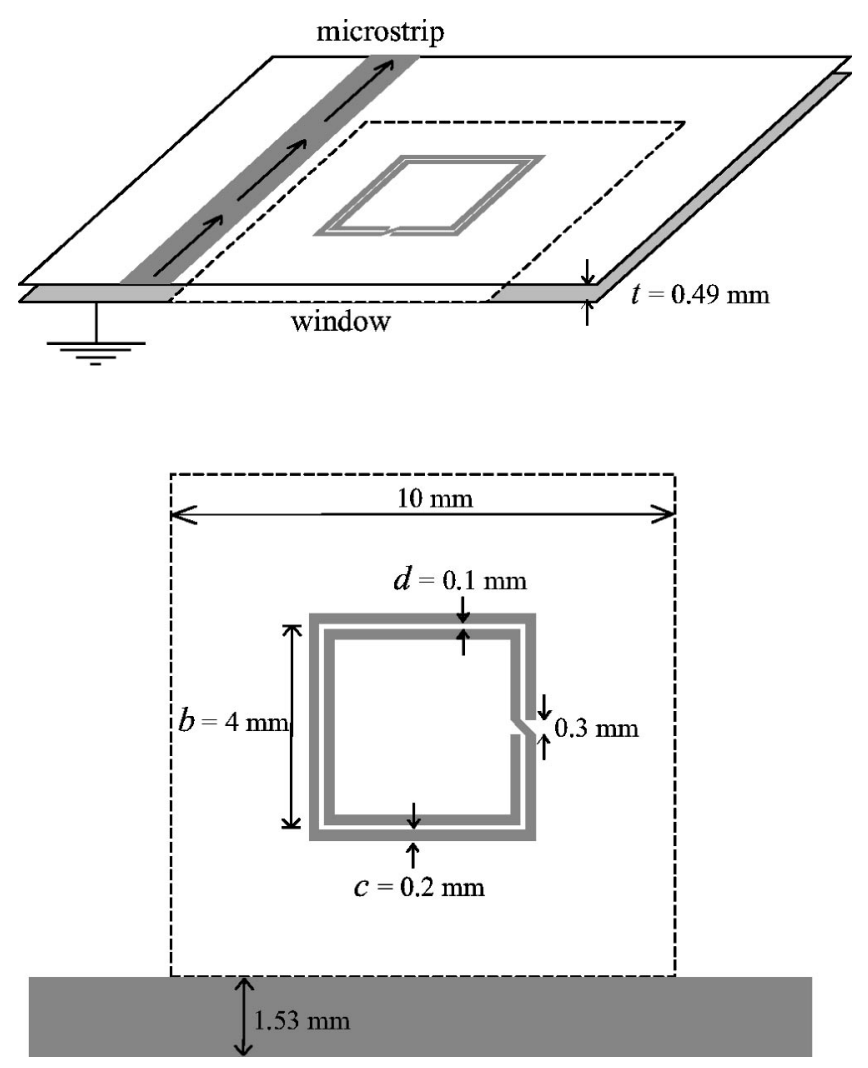

FIG. 2. The setup used for the numerical simulations. The relative dielectric constant of the substrate $\epsilon_{r}=2.43$. Metallizations of copper with a thickness of $35 \mu \mathrm{m}$ are used.
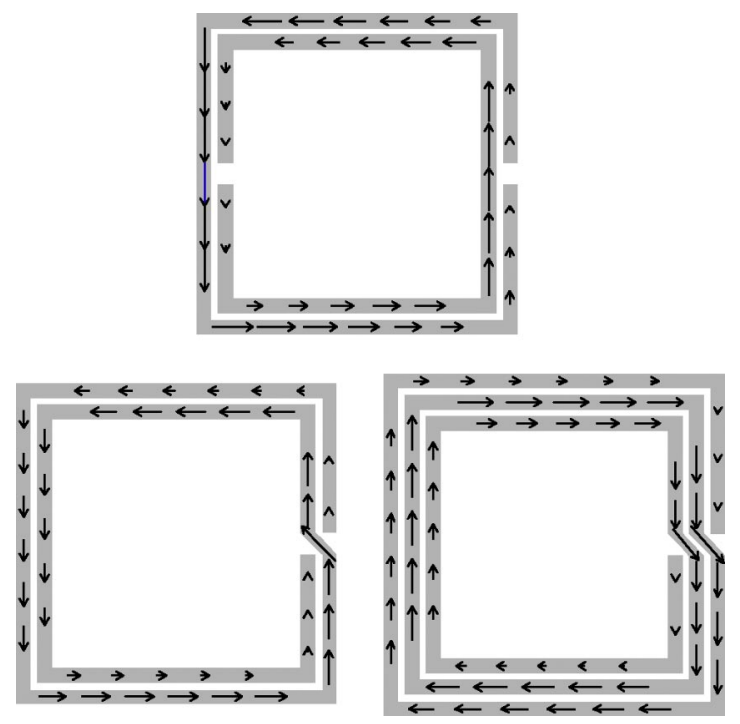

FIG. 3. Distribution of electric current density on the particles when the resonant mode is excited. The geometrical parameters are shown in Fig. 2. As it can be seen in the figure, the mean perimeter for the three square rings forming SR3 is the same as for the two rings forming SRR or SR2.

polarizabilities. ${ }^{5}$ However, the symmetrical distribution of the electric field between the strips of the SR's clearly suggests that a magnetic excitation cannot generate an electric dipole in the particle. Therefore, in the quasistatic limit cross polarization effects are not expected in SR's. This fact could be of interest in the realization of isotropic metamaterials. To end this section, it can be noted that, from the circuit models presented in Fig. 1, the magnetic moment associated with the SR's, $m_{x} \approx \pi r_{0}^{2} I$, is given by

$$
m_{x}=\alpha_{x x}^{m m} B_{x}^{\mathrm{ext}}, \quad \alpha_{x x}^{m m} \approx \frac{\pi^{2} r_{0}^{4}}{L}\left(\frac{\omega_{0}^{2}}{\omega^{2}}-1\right)^{-1},
$$

where $r_{0}$ is the mean radius of the particle.

\section{VALIDATION OF THE MODEL}

In order to verify the proposed hypothesis about the current distribution at resonance on the SRR and on the SR's, a simulation has been carried out by using the commercial package ENSEMBLE (this software performs the frequency domain analysis of planar printed structures useful in microwave integrated circuit and printed antenna applications; a smart method of moments algorithm is implemented in it). The square geometry shown in Figs. 2 and 3 has been used for simplicity in computations. We feel, however, that the conclusions can be generalized to other geometries. Each resonator was excited by a conventional microstrip line located close to and at the same plane as the resonant particle. A window was opened in the ground plane in the region occupied by the rings in order to not meaningfully perturb the magnetic-field configuration of the particle under study. The results of these simulations are summarized in Fig. 3 and in Table I. The length of the arrows in Fig. 3 is proportional to the current amplitude on each ring. It can be seen that the 
TABLE I. Comparison among the resonance frequencies provided by electromagnetic simulation and approximate quasistatic formulas for the structures in Fig. 3.

\begin{tabular}{lcc}
\hline \hline & $f_{0}^{\text {sim }}(\mathrm{GHz})$ & $f_{0}^{\text {th }}(\mathrm{GHz})$ \\
\hline SRR & 4.82 & 4.70 \\
SR2 & 2.46 & 2.35 \\
SR3 & 1.64 & 1.66 \\
\hline \hline
\end{tabular}

current distribution shown in Fig. 3 qualitatively agrees very well with the proposed model description (see current distributions in Fig. 1). The results for the resonance frequencies of each particle (shown in Table I) confirm the rule in Eq. (3). The theoretical frequencies in Table I were obtained from the model proposed in section II for circular particles having the same area $\left(\pi r_{0}^{2}=b^{2}\right)$ as the square particles in Fig. 3. Therefore, extremely accurate agreement cannot be expected. However, the reasonably good agreement between both set of frequencies shows the adequacy of the proposed model (quasistatic in nature) and illustrates the advantages of the spiral resonators associated with their small size.

Experiments have been also carried out to check the model. The frequency of resonance of several SRR and SR's have been measured. For this purpose, each particle was placed in the central $E$ plane of a rectangular waveguide excited in its fundamental $\left(\mathrm{TE}_{01}\right)$ mode. The transmission coefficient $\left(\left|S_{12}\right|\right)$ was measured by means of the automatic network analyzer HP-8510-B. An absorption dip was observed for every particle at the resonance frequency. The experimental results shown in Table II confirm once again the rule (3), also showing a good quantitative agreement with the simple theory developed in Sec. II.

\section{DESIGN OF METAMATERIALS USING SR2 PARTICLES}

In the preceding section the validity of the model proposed for the analysis of the SR's has been demonstrated. In this section, the usefulness of the SR's for designing artificial LHM and NMPM will be shown. With this goal in mind, the experimental procedure reported in Ref. 6 has been adapted for this purpose (see Fig. 4). An artificial LHM is simulated by a regular array of SR's placed at the half plane of a host square $\left(6 \times 6 \mathrm{~mm}^{2}\right)$ waveguide, which is excited in its fundamental mode. The spirals were printed on a microwave

TABLE II. Comparison among the resonance frequencies provided by experiment and elementary quasi-static theory. The structures are the ones shown in Fig. 1 with $r_{0}=2.45 \mathrm{~mm}, c$ $=0.3 \mathrm{~mm}, d=0.5 \mathrm{~mm}$. They are printed on a dielectric substrate (ARLON CuClad 233LX) with $\epsilon_{r}=2.33$ and thickness $0.127 \mathrm{~mm}$. Metallizations are of copper with a thickness of $35 \mu \mathrm{m}$.

\begin{tabular}{lcc}
\hline \hline & $f_{0}^{\exp }(\mathrm{GHz})$ & $f_{0}^{\text {th }}(\mathrm{GHz})$ \\
\hline SRR & 6.15 & 6.18 \\
SR2 & 3.24 & 3.09 \\
SR3 & 2.23 & 2.18 \\
\hline \hline
\end{tabular}

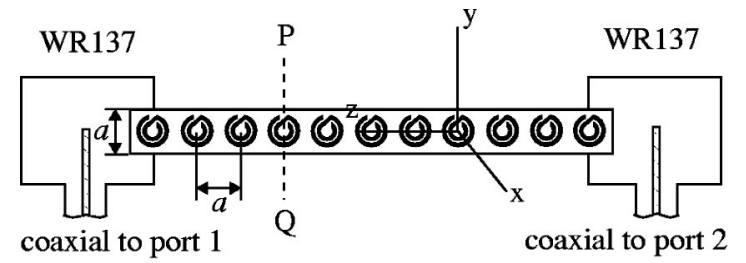

a)

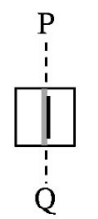

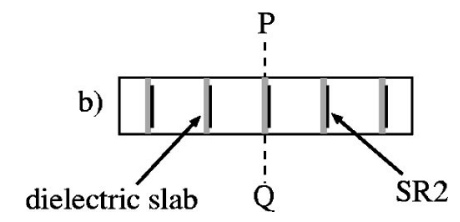

FIG. 4. Sketch of the experimental setup used to illustrate NMPM and LHM simulation. A SR-loaded rectangular waveguide is placed between two commercial coaxial and rectangular waveguide junctions. Two SR-loaded waveguide sections are used in order to simulate a LHM (a) and a waveguide filled by a NMPM (b).

substrate (ARLON CuClad 250LX) with dielectric thickness $0.49 \mathrm{~mm}$, dielectric constant $\epsilon_{r}=2.43$, and metallization thickness $35 \mu \mathrm{m}$. The geometrical dimensions of the printed SR's are external radius $r_{\text {ext }}=2.1 \mathrm{~mm}$, width of the strips $c$ $=0.5 \mathrm{~mm}$, and distance between strips $d=0.3 \mathrm{~mm}$. Eleven SR's were aligned forming a row inside a 60 -mm-long waveguide section [see Fig. 4(a)]. The measured frequency of resonance for the individual spirals was $f_{0}=5.4 \mathrm{GHz}$. Since the host waveguide cutoff is at $\approx 25 \mathrm{GHz}$, the theory ${ }^{6}$ predicts a LHM pass band just above the frequency of resonance of the SR's. This prediction is fully confirmed by the experiment, as it is seen in Fig. 5.

A similar experiment was made by replacing the square waveguide of Fig. 4(a) by a rectangular waveguide $30 \mathrm{~mm}$ wide [see Fig. 4(b)]. This waveguide was loaded with five equally spaced identical SR2 rows, each row having 11 spiral rings. Note that the cutoff frequency of the rectangular waveguide is now below the resonance frequency of the SR's. Thus, the whole device is a reasonably good simulation of a

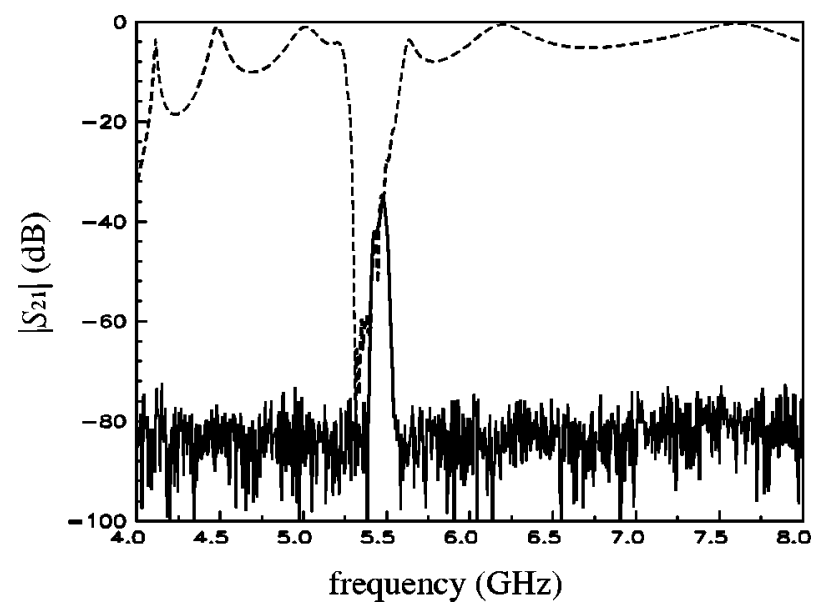

FIG. 5. Measured transmission coefficients through the SR'sloaded waveguides in Fig. 4. Solid lines: passband in the SR-loaded square waveguide of Fig. 4(a). Dashed lines: stop band in the SRloaded rectangular waveguide of Fig. 4 (b). 
NMPM in the range of frequencies immediately above the resonance frequency of the individual rings. It can be considered, in fact, as a rectangular waveguide filled by an anisotropic NMPM with $\mu_{x x}<0$, similarly as in Refs. 10 and 11 Since the waveguide is above cutoff in the frequency range where the effective permeability of the medium filling the guide is negative, a stop band is expected in this frequency range. This prediction is confirmed by the experiments, as it is clearly shown in Fig. 5. It is worth to note that the upper limits of the pass band and the stop band exactly coincide, which is an indirect confirmation of the nonbianisotropic behavior of the simulated LHM and NMPM. ${ }^{5}$ The rejection band starts slightly before than the passband. This fact can be explained by the presence of a region of anomalous dispersion with high losses around the frequency of resonance of the individual SR's. In this region no measurable transmission is expected neither for the NMPM nor for the LHM.

\section{CONCLUSIONS}

The usefulness of planar spiral resonators (SR's) for the design of artificial discrete magnetic media, including negative magnetic permeability media (NMPM) and left-handed media (LHM), has been shown at microwave frequencies by both theory and experiment. The proposed particle has sev-

*Electronic address: juan_dbd@us.es

†Electronic address: marques@us.es

†Electronic address: medina@us.es

${ }^{\S}$ Electronic address: martel@us.es

${ }^{1}$ J.B. Pendry, A.J. Holden, D.J. Robbins, and W.J. Stewart, IEEE Trans. Microwave Theory Tech. 47, 2075 (1999).

${ }^{2}$ D.R. Smith, W.J. Padilla, D.C. Vier, S.C. Nemat-Nasser, and S. Schultz, Phys. Rev. Lett. 84, 4184 (2000).

${ }^{3}$ V.G. Veselago, Sov. Phys. Usp. 10, 509 (1968).

${ }^{4}$ W. Rotman, IRE Trans. Antennas Propag. 10, 82 (1962).

${ }^{5}$ R. Marqués, F. Medina, and R. Rafii-El-Idrissi, Phys. Rev. B 65, 144440 (2002).

${ }^{6}$ R. Marqués, J. Martel, F. Mesa, and F. Medina, Phys. Rev. Lett. eral advantages over other previously proposed resonators, such as the split ring resonator $(\mathrm{SRR})^{1}$ or the modified broadside coupled SRR (BC-SRR). ${ }^{5}$ First of all SR's are easy to manufacture using well-known and relatively cheap technologies (photo-etching, for instance), because of its uniplanar character and the absence of the necessity of fabricating narrow slots between the strips or other fine details in the etched design. Moreover, the use of SR's allows for a significant potential reduction in the electrical size of the metamaterial unit cell when compared with other alternatives. This last property has special significance if the metamaterial has to be described as a continuous medium rather than as a discrete periodic structure. Finally, SR's retain the nonbianisotropic behavior of other proposed structures - such as the aforementioned BC-SRR-its manufacturing and the control of its electrical properties being considerably easier. This feature is of interest in the design of isotropic metamaterials for various applications, such as negative refraction or subwavelength focusing experiments.

\section{ACKNOWLEDGMENTS}

This work was supported by the Spanish Ministry of Science and Technology and FEDER, under the CICYT Project No. TIC2001-3163.

89, 183901 (2002).

${ }^{7}$ R. Marqués, J. Martel, F. Mesa, and F. Medina, Microwave Opt. Technol. Lett. 35, 405 (2002).

${ }^{8}$ I. Bahl, and P. Bhartia, Microwave Solid State Circuit Design (Wiley, New York, 1988).

${ }^{9}$ R. Marqués, F. Mesa, J. Martel and F. Medina, in Metamaterials, special issue of comments IEEE Trans. Antennas Propag. 51, 2572 (2003).

${ }^{10}$ J. Baena, R. Marqués, J. Martel, and F. Medina, IEEE AP-S International Symposium, Columbus, Ohio, 2003 (CD-ROM of the Symposium).

${ }^{11}$ S. Hrabar and J. Bartolic, IEEE AP-S International Symposium, Columbus, Ohio, 2003 (CD-ROM of the Symposium). 